\title{
Entrevista a Mirion Malle
}

\section{Interview avec Mirion Malle}

\section{Por Emma Le Lain Per Emma Le Lain}

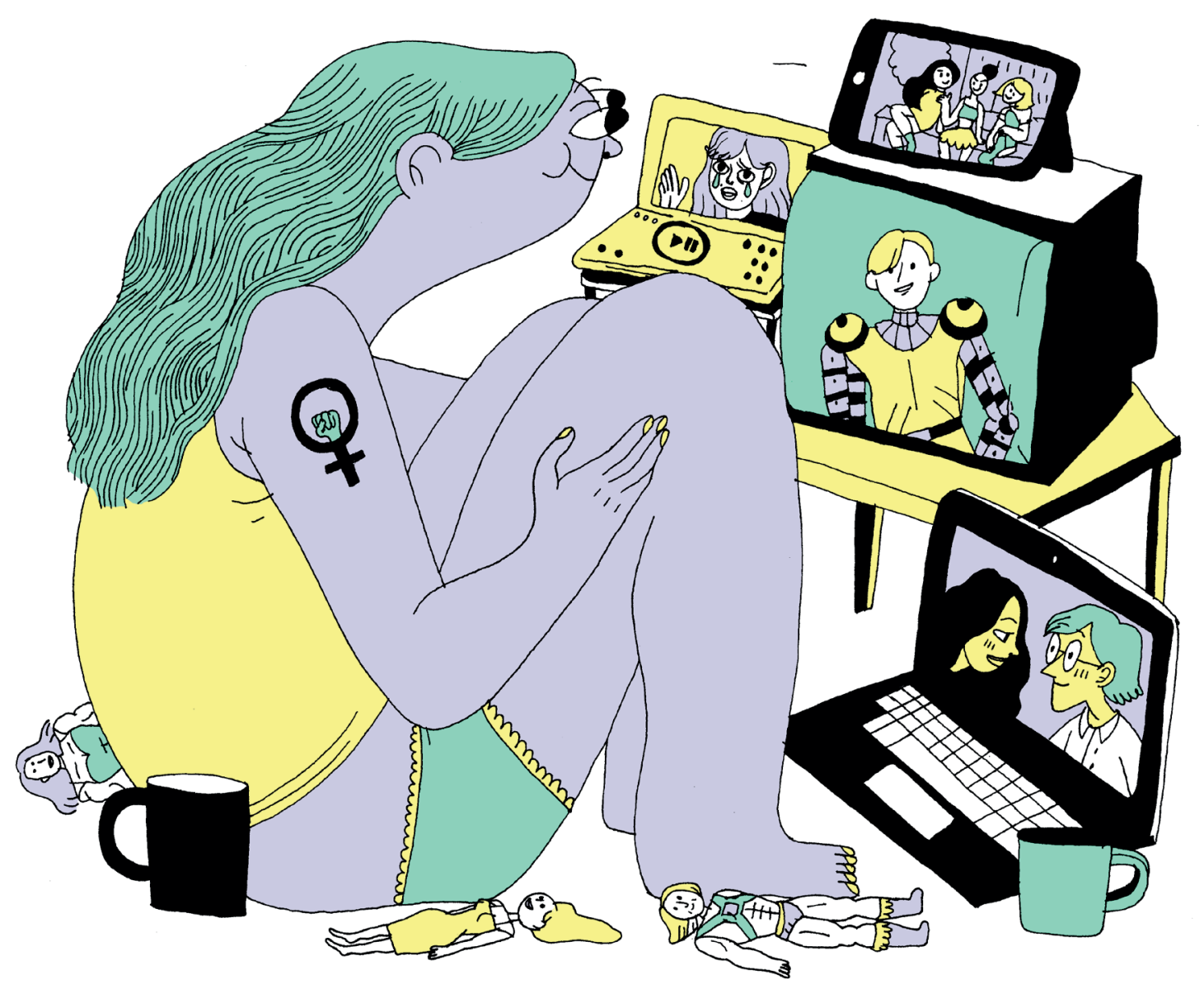

Imagen: Comando Culotte. París, Ankama, 2016. Portada 


\section{CuCoEntrevista}

La siguiente entrevista se ofrece tanto en el francés en el que Emma Le Lain la realizó originalmente como en su traducción al castellano.

La entrevista se realizó mediante correo electrónico en el mes de abril de 2016, con la amabilidad y el entusiasmo de Mirion por dar a conocer su trabajo.

Marion Mille, conocida en el mundo del cómic como Mirion Malle, es una autora francesa de 24 años que acaba de publicar en Francia su primera obra, Commando Culotte (Ankama, 2016), tras varios años de éxito en su blog del mismo título. ${ }^{1}$ Como muchos autores de su generación, participó en la ola de autopublicación que apareció en Francia gracias a los blogs. Este soporte virtual de fácil acceso se desarrolló rápidamente en torno a los años 2008 y 2009, lo que permitió a mucha gente, dibujantes de oficio o no, expresarse y compartir sus ideas a través del cómic, como fue el caso de Mirion. Así nació, de repente, una multitud de voces en soportes virtuales, lo que se convirtió en un auténtico crisol de opiniones con reacciones diversas por parte del público.

En este contexto surgió Commando Culotte, cuyo trabajo se centra en el feminismo y en la representación, unos temas que le interpelaron por su propia experiencia hasta constituir el eje principal de su blog.

${ }^{1}$ http://www.mirionmalle.com/
Mirion a répondu à notre interview à propos de son travail par mail, au mois d'avril 2016, avec amabilité et enthousiasme.

Marion Mille, connue dans le monde de la BD comme Mirion Malle, est une autrice française de 24 ans qui vient de publier son premier livre en France, Commando Culotte (Ankama, 2016), après plusieurs années de succès sur son blog du même nom. ${ }^{1}$ Comme bon nombre d'auteurs de sa génération, elle a participé à la vague d'autopublication qui est apparue en France avec les blogs. Ce support virtuel facile d'accès a en effet connu un essor vers 2008-2009, ce qui procura à tous une visibilité supplémentaire et un moyen de se faire connaître via son travail, que l'on soit dessinateur de métier ou, comme Mirion, désireux de partager et de s'exprimer à travers la BD. Ainsi, une foule de coups de crayon se sont fait connaître, jusqu'à devenir un véritable creuset d'opinions et de réactions de la part du public.

C'est dans ce contexte qu'est apparu Commando Culotte, dont le travail se centre sur le féminisme et la représentation: des questionnements personnels qui sont devenus

${ }^{1}$ http://www.mirionmalle.com/ 
Así, conforme iba proponiendo elementos de reflexión a través de su llamativo dibujo empezó a analizar productos culturales, principalmente series y películas anglófonas, francófonas, pero también japonesas y de otros países. En estos análisis destaca no solo temas relativos a las mujeres sino también muchos otros temas transversales: leyendo a Mirion Malle se encuentran pistas para reflexionar a propósito del racismo, el clasismo, nuestra relación con los medios de comunicación y sus consecuencias en la educación. ¡Sin olvidar su siempre presente amor por Jean-Claude Van Damme y la pelea! Esta vertiente de su cómic le permitió hacerse conocer en varios medios de comunicación online franceses, que en muchas ocasiones se ocupan también del feminismo. A través de su trabajo, Mirion se inscribe en esta corriente de vulgarización vigente en el Internet francófono, que nos da a entender por qué, al fin y al cabo, cada objeto cultural es político.

Además de todo ello, Mirion estudió didáctica del cómic en una escuela belga, lo que ha hecho más interesante la propuesta de entrevista para que nos explique su trabajo y algunas opiniones sobre el mundo del comic francófono.

\section{Hola, Mirion, ¿nos puedes resumir tu re- corrido como estudiante y autora?}

Bueno, primero hice un año preparatorio de literatura con opción de teatro en Toulouse, luego un tercer año de grado en estudios teatrales en la Sorbonne en $\mathrm{Pa}$ rís, luego un grado de Cómic en el ESA St Luc en Bruselas, y por fin un posgrado de sociología especializado en género, de nuevo en París. Y paralelamente empecé un blog en 2011, hice varias autopublicaciones junto con otros autores, $y$ ahora l'axe principal de son blog. Dès lors, elle nous a proposé des éléments de réflexion à travers de son dessin accrocheur puis s'est mise à analyser des objets culturels, principalement des films séries et séries anglophones, francophones, mais aussi japonais et d'autres pays encore. Dans ces analyses, elle aborde le féminisme mais également des thèmes transversaux: en lisant Mirion, on trouve des pistes de réflexion sur le racisme, le mépris de classe, notre rapport aux médias et ses conséquences sur l'éducation. Sans oublier son amour toujours présent pour Jean-Claude Van Damme et la bagarre! C'est ce pan de son travail qui lui a permis de se faire connaître par d'autres médias français en ligne, souvent axés eux aussi sur le féminisme. Via son œuvre, Mirion Malle s'inscrit dans cette dynamique actuelle de l'Internet francophone, la vulgarisation; celle qui nous permet de comprendre pourquoi, au bout du compte, tout objet culturel est aussi politique.

En outre, Mirion a étudié la didactique de la $\mathrm{BD}$ dans une école belge, un pan intéressant de son parcours qu'elle va nous expliquer ici, tout en nous donnant quelques opinions à propos du monde de la bande dessinée.

Salut Mirion, peux-tu nous résumer ton parcours d'étudiante et d'autrice?

Alors, j'ai tout d'abord fait une prépa littéraire option théâtre à Toulouse, puis une 3eme année de licence d'Etudes Théâtrales à la Sorbonne, puis un baccalauréat (l'équivalent d'une licence) en Bande Dessinée à l'ESA St Luc à Bruxelles, et enfin un master de sociologie spécialité genre à Paris à nouveau. Et en parallèle, j’ai commencé un blog en 2011, fait plusieurs autopublications en groupe, et là je viens de sor- 
acabo de estrenar una adaptación del blog en la editorial Ankama. ¡Uf! Estar en la escuela Saint Luc en Bruselas en el grado de cómic me ayudó mucho. Me dio muchas técnicas que me hubiera costado aprender sola: cómo componer las viñetas, las páginas, cómo poner algo de manifiesto, cómo construir un escenario, o tan solo algunas técnicas como el «pay-in / pay-out» (es decir, utilizar de nuevo un elemento que ha sido introducido antes en la historia). Es útil, y sobre todo dibujamos cómics constantemente durante tres años, lo que lleva forzosamente a mejorarse. Sin embargo, ¡no es algo imprescindible ir a una escuela! Puede ser un medio útil para adquirir técnicas y encontrarse con la gente, pero para mí lo importante es escribir, dibujar, leer un montón de cómics y también enseñar el resultado a los demás pidiéndoles que te critiquen. Además, se puede decir que la estructura de una escuela no conviene a todo el mundo, muchas veces uno no puede hacer lo que le da la gana, y contrariarse no sirve para nada. Con Internet ahora es también más fácil integrarse en una red de personas que hacen cómic, y que, de hecho, te pueden dar opiniones un poco más precisas.

\section{Tu trabajo en Internet, como tu último libro publicado por Ankama, se enfoca principalmente en la representación. ¿A1- gunas historias que compartir en cuanto a la recepción de tus cómics?}

Está claro que hacer feminismo en Internet conlleva un montón de reacciones diversas. Por supuesto, mucho odio, rechazos, insultos... Bueno, una se acostumbra a esto, aunque siempre es bastante desagradable; pero recientemente se trata, sobre todo, de reacciones de interés, de personas feministas o que se están abriendo al feminismo, y de crí- tir une adaptation du blog chez Ankama! Ouf! J'étais en section BD à St-Luc et ça m’a beaucoup aidée. En fait, ça m’a donné beaucoup de techniques que j'aurais eu du mal à acquérir seule: comment composer mes cases, comment composer mes pages, comment mettre quelque chose en évidence, comment construire un scénario, ou plus simplement des techniques comme le «payin / pay-out» (réutiliser un élément qui a déjà été présenté plus tôt dans l'histoire). C'est utile, et surtout comme on dessine et qu'on fait des $\mathrm{BD}$ constamment pendant trois ans, on est obligés de progresser. Faire une école, après, c'est loin d'être nécessaire! Ça peut être pratique pour acquérir des techniques et rencontrer des gens, mais je pense que l'important c'est d'écrire et de dessiner, de lire BEAUCOUP de bandes dessinées, et aussi de montrer son travail et de demander des critiques. En plus, le format école ne peut pas convenir à tout le monde, c'est très scolaire, on ne peut pas forcément faire ce qu'on a envie, et ça ne sert à rien de se forcer. Aussi, avec internet, maintenant c'est plus facile de «rentrer» dans un réseau de gens qui font de la BD également et qui du coup peuvent donner des avis un peu plus précis!

Ton travail visible sur Internet, tout comme ton dernier livre publié chez Ankama, se centre principalement sur la représentation. Des anecdotes à partager sur la réception de tes notes de blog?

Faire du féminisme sur Internet ça amène forcément tout un tas de différentes réactions. Bien sûr beaucoup de haine, de rejets, d'insultes, bon, on s'y fait, même si c'est toujours pas mal désagréable; mais ces derniers temps, c'est surtout de l'intérêt, des personnes féministes ou qui s'ouvrent au féminisme et des critiques constructives ou 
ALORS CERTES ON PEUT VOIR DES DIFFÉRENCES ENTRE LES PERSONNAGES...


DÉJĀ, POUR DONNER DES MODÉLES ÁTOUT LE MONDE.
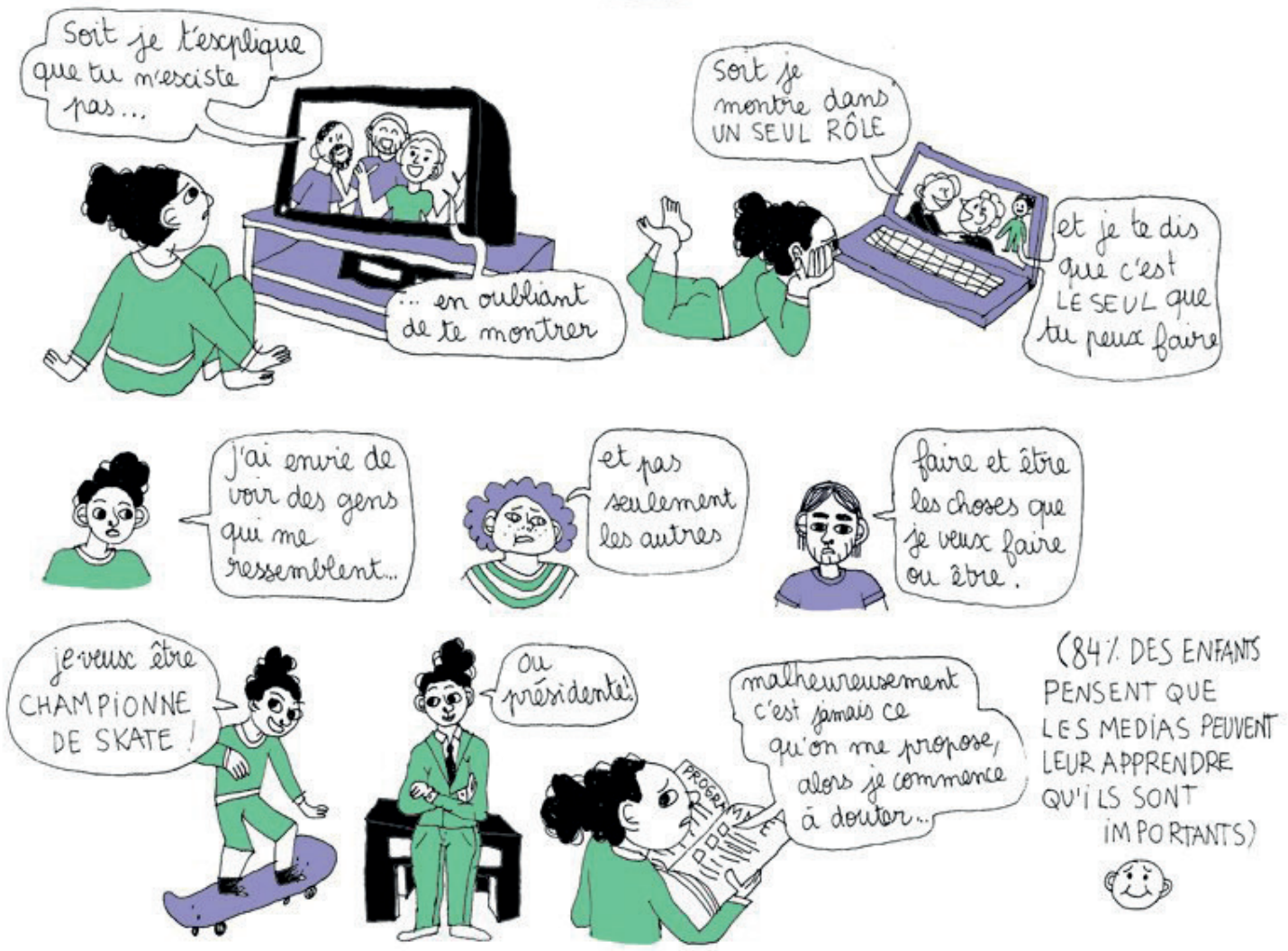

Mirion explica que es importante dar a los niños varios modelos que no sean dependientes de su sexo.
Mirion explique ici en quoi il est important de donner aux enfants des modèles variés, non reliés à leur genre. 
ticas constructivas o intercambio de páginas web, series, pelis... Vamos, ¡que eso está bien!

\section{¿Piensas en vincular tu posgrado en género con tus futuros proyectos de cómic? ¿Cómo construyes tus cómics pedagógicos?}

Sí, tengo un proyecto relacionado con el feminismo, que supone una encuesta sociológica, iy me gustaría mucho empezar! Seguro que lo haré después del posgrado.

En cuanto a mis cómics, encuentro el tema, paso por un primer momento de reflexión, leyendo, informándome, y después hago un plan, como para un ensayo. Luego dibujo directamente, me parece que así la construcción y la lectura son más claras.

\section{Tus cómics ponen de realce las luchas in- terseccionales, ${ }^{2}$ se aprende mucho. ¿Có- mo te fuiste sensibilizando hacia estas ideas?}

¡Twitter! Es un medio de comunicación maravilloso que me permitió seguir a muchas feministas distintas y salir de este feminismo blanco, que hasta entonces veía y conocía más que otra cosa. Por ejemplo, empecé por seguir a @Mar_Lard (¡que prologó mi libro!), que publica mucho a propósito de los videojuegos e hizo dos artículos muy buenos sobre este tema. Desde entonces las militantes feministas constituyen casi el 80\% de mi timeline: @femfreq (Anita Sarkeesian, de la cadena Feminist Frequency), @A_C_Husson (que tiene un blog a propósito del género, Genre!),3 @The_Economiss

${ }^{2} \mathrm{E}$ l feminismo interseccional es una corriente que lucha contra las discriminaciones de varios tipos conjugadas: el color de piel, la procedencia social, la no-heterosexualidad, la obesidad, etc.

${ }^{3}$ https://cafaitgenre.org/ du partage, de liens ou de séries, de films. Bref, c'est plutôt chouette!

Penses-tu relier ton master Genre avec tes futurs projets en $\mathrm{BD}$ ? Comment construis-tu tes notes pédagogiques (vulgarisation...)?

Oui, j'ai notamment un projet, toujours en rapport avec le féminisme, qui demande un travail de terrain sociologique et $j$ 'ai vraiment hâte de m'y mettre, mais du coup ce sera probablement après mon master!

Et pour mes notes, je trouve le sujet, je fais un petit brainstorming, je lis, je me renseigne, puis je fais un plan, comme pour une dissertation. Ensuite, je dessine directement, je trouve que ça permet une construction et une lecture plus fluide!

Tes BD donnent une belle place aux luttes intersectionnelles, ${ }^{2}$ on y apprend beaucoup. Comment as-tu été sensibilisée à ces idées?

Twitter! C'est un outil merveilleux qui m'a permis de suivre tout un tas de féministes différentes et de me sortir de ce féminisme blanc que je connaissais et voyais surtout jusque-là. Par exemple, une des premières que j'ai suivi, c'est @Mar_Lard (qui a fait la préface de mon livre, hihi), qui tweete beaucoup sur le jeu vidéo et qui a fait deux excellents articles sur le sujet, mais depuis les militantes féministes constituent presque $80 \%$ de ma timeline: @femfreq (Anita Sarkeesian,de la chaîne Feminist Frequency), @A_C_Husson (qui tient un blog sur le genre, Genre!), ${ }^{3}$ $@$ The_Economiss (qui parle de finance et

${ }^{2}$ Le féminisme intersectionnel lutte contre les discriminations conjuguées: la couleur de peau, la classe sociale, la non-hétérosexualité, l'obésité, etc.

${ }^{3}$ http://çafaitgenre.org 


\section{Rendre L Emiman Postif}

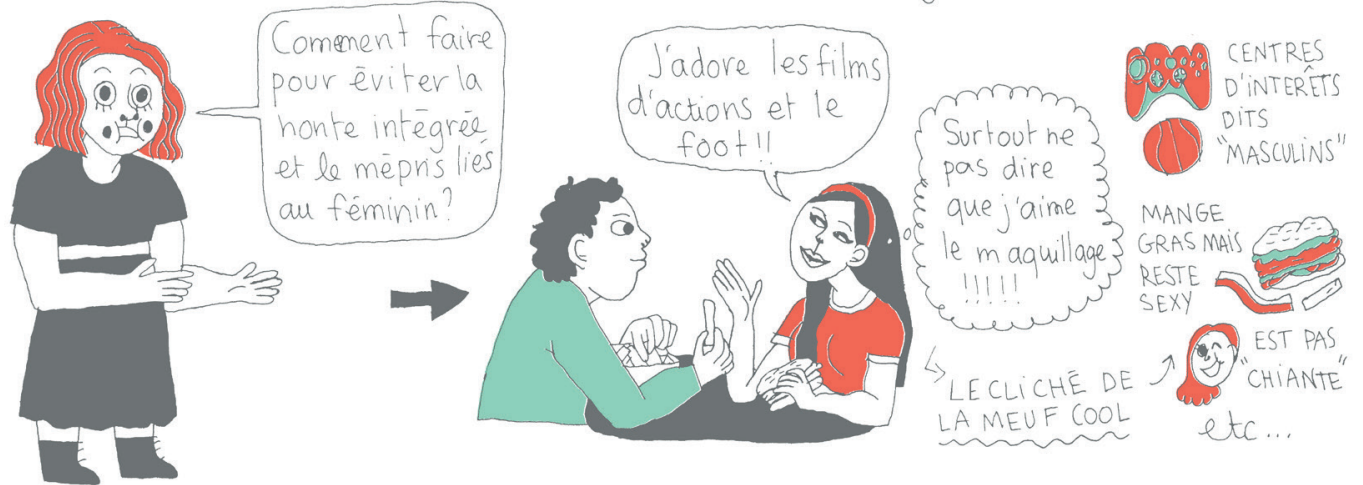

$\hookrightarrow$ IL FAUT RËHABILITER LES CARACTÉRISTIQUES "FÉMININES".
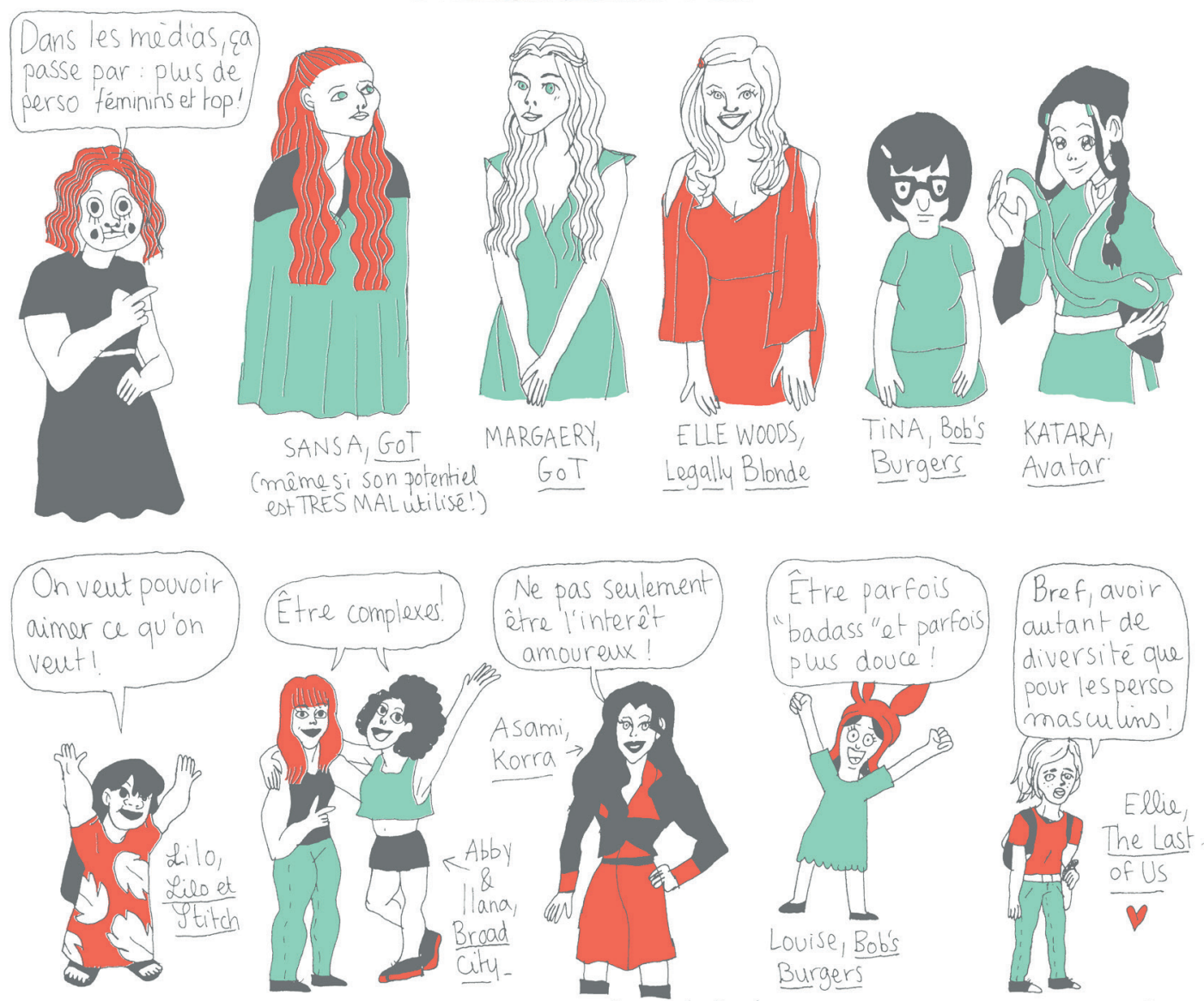

$\rightarrow$ BREF. ON VEUT DES PERSONNAGES FEMININS DIVERS, AVEC DES CARACTERISTIQUES

"FÉMININES" COMME "MASCULINES", MÉLANGEES OU NON.

Convertir lo femenino en algo positivo rehabi-

litando sus características: que saber pelear sea tan importante como saber cuidar.
Faire du «féminin» un élément positif en rébabilitant ses caractéristiques: savoir se battre devrait être aussi important que de savoir prendre soin des autres. 
(que habla de finanzas y de afrofeminismo), @ComicSansInes (que tuitea sobre feminismo y antirracismo), iy muchos más! Es muy instructivo, y muchas iniciativas nacen en Twitter. Por ejemplo, para el 8 de marzo de este año participé en @Radiofeministe_ (radio feminista), una cuenta compartida en la que intervenimos en grupo en diferentes temas, como si fueran conferencias, ipero en Twitter! Yo hablé de feminismo y cómic, pero fue genial poder leer a otras feministas a propósito de muchos temas importantes.

\section{A tu parecer, ¿cómo estamos con la difu- sión de las ideas feministas y la represen- tación en Francia? ¿Comprobaste algunas diferencias con respecto a otros países?}

Por haber vivido en Bélgica, me parece que la sensibilización es un poco mayor que en Francia, pero tampoco es radicalmente distinta. También estuve cuatro meses en Montreal, donde efectivamente vi una gran diferencia con Francia: casi no existe la violencia sexista en las calles, la mayoría de la gente está sensibilizada con el feminismo, hay menos rechazo, más representación, eventos feministas... Pero al mismo tiempo soy consciente de que allí estuve en un medio particular: el del cómic. Así que no creo que sea la única realidad. Pero si, por ejemplo, se compara con el mundo del cómic en Francia, es diferente de verdad.

De todas formas, me parece que aquí en Francia, tenemos retraso en este tema, hay tanta gente que hace todo lo que puede para evitar que cambien las cosas, es una pena...

\section{¿Qué tendencias te parecen más represen- tativas del feminismo francés en el cómic actual?}

No pienso que pueda dar una lista buena de verdad. Me parece que cada vez más autoras d'afro-féminisme), @ComicSansInes (qui tweete féminisme et anti-racisme) et beaucoup d'autres encore! C'est tellement instructif, et beaucoup d'initiatives naissent sur Twitter! Par exemple, pour le 8 mars cette année, j’ai participé à @Radiofeministe_, un compte commun où on est intervenues à plusieurs sur différents sujets, comme un colloque et ses conférences, mais sur Twitter! J'ai parlé féminisme et $\mathrm{BD}$, et c'était vraiment bien de pouvoir lire d'autres féministes sur tout un tas de sujets importants.

Où en est, selon toi, la diffusion des idées féministes et sur la représentation en France? As-tu constaté des différences avec d'autres pays?

Pour avoir vécu en Belgique, je trouve qu'il y a une sensibilisation un peu plus grande qu'en France mais pas non plus radicalement différente. J'ai aussi été quatre mois à Montréal, où là effectivement $\mathrm{j}$ 'ai senti un gros décalage avec la France: quasiment pas de harcèlement de rue, la plupart des gens sensibilisés au féminisme, moins de rejet, plus de représentation, d'événements féministes... Mais je suis aussi consciente que là-bas j'ai évolué dans un milieu particulier, celui de la BD, donc je ne cherche pas à donner une vérité générale. Mais par exemple, si on compare au monde de la BD en France, c'est vraiment différent.

Je pense que de toute façon on est vraiment en retard sur ce sujet ici, il y a tellement de gens qui mettent tout en œuvre pour éviter que ça change, c'est affligeant...

Quelles sont à ton avis les tendances les plus représentatives du féminisme français dans la bande dessinée actuelle?

Oh, je ne pense pas que je pourrais donner une vraie bonne liste. J'ai l'impression que 


\section{Elingue on Rouge-à-Lères:}

UN PERSONNAGE FEMMININ RÉUSSIT DOIT-IL FORCEMENT ÊTRE VIRIL?
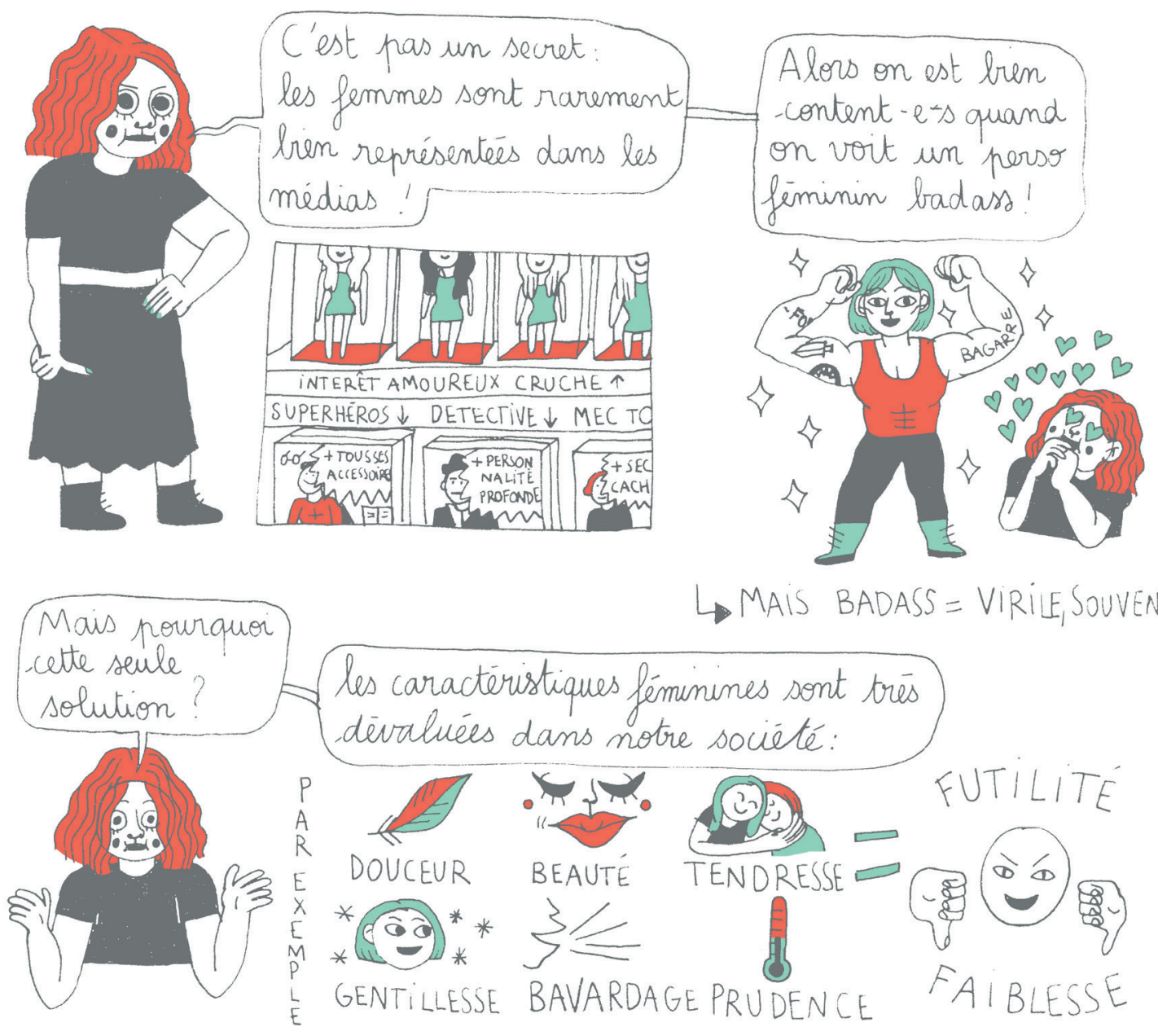

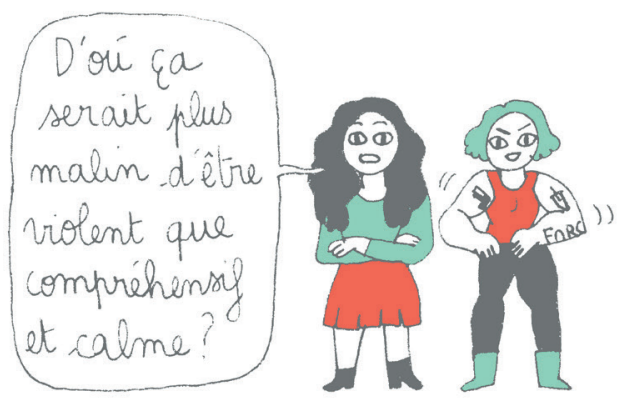

¿Pistola o pintalabios? ¿Debe ser viril un personaje femenino para ser bueno?

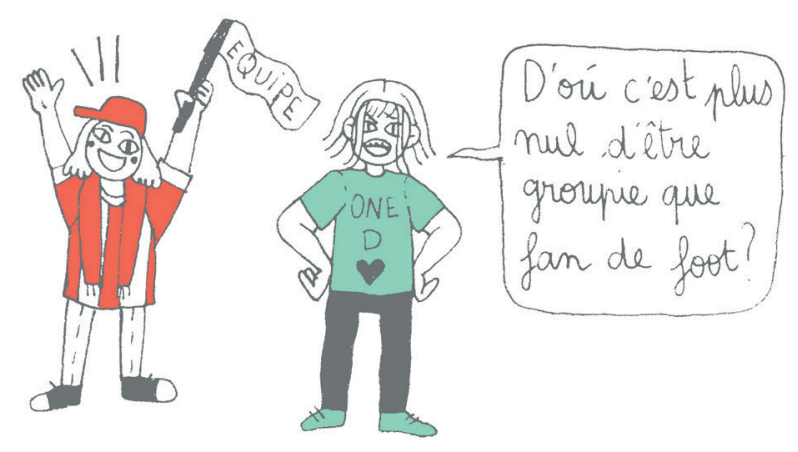

Flingue ou rouge à lèvres? Un bon personnage féminin doit-il obligatoirement être viril? 
- $\mathrm{y}$ algunos autores - se reivindican como feministas y eso es genial. ¡Pero creo que diría las firmantes del Colectivo de Creadoras! ${ }^{4}$

\section{Justamente formas parte del Colectivo de Creadoras de Cómic contra el Sexismo. ¿Nos podrías explicar tu trayectoria en esta estructura?}

No hice tantas cosas, al principio recibí un correo que hablaba de esto, me informé y firmé rápidamente, porque me parecía una iniciativa saludable. Después estuve un poco más activa en torno al caso del Salón de Cómic de Angoulême de este año, primero en las redes sociales y luego participé en el evento del Colectivo durante el festival. Está bien que exista el Colectivo, nos parece que somos más fuertes y que por fin nos escuchan un poco, aunque no basta con eso, por supuesto.

\section{En enero de este año se habló mucho de} la polémica de los treinta autores nombrados en el Grand Prix de Angoulême, sin que haya una sola autora en esta lista. ¿Un atraso puntual o un reflejo directo de la situación de las mujeres en el mundo del cómic actual?

Es un reflejo directo en el sentido de que están constantemente puestas en segundo

${ }^{4}$ El Colectivo de Creadoras de Cómic contra el Sexismo es un conjunto de autoras que en diciembre de 2013 se reunieron indignadas por ser consideradas mujeres antes que autoras de cómic. Organizaron varios eventos como el paródico «Los hombres y el cómic». Aunque la idea común en el mundo del cómic francés es que existen muy pocas autoras, las firmantes del Colectivo son más de doscientas, y tuvieron un papel destacable en el escándalo del Gran Premio de Angoulême en enero de 2016. El Colectivo tiene una página web, http:// bdegalite.org/, y publica artículos con regularidad. La versión en español: http://bdegalite.org/english/ carta-de-autoras/ de plus en plus d'autrices - et un peu des auteurs - se revendiquent féministes et c'est vraiment top. Mais je pense que je dirais les signataires du Collectif des Créatrices! ${ }^{4}$

Justement, tu fais partie du Collectif des Créatrices de Bande Dessinée contre le Sexisme. Peux-tu nous résumer ton parcours au sein de cette structure?

Je n'ai pas fait grand-chose, au début,j'ai reçu un mail qui en parlait, je me suis renseignée et j’ai très vite signé, parce que ça me paraissait salutaire comme initiative. Ensuite, j'ai été un peu plus active autour d'Angoulême, sur les réseaux sociaux d'abord puis j’ai participé à l'événement du Collectif à l'occasion du festival. C'est tellement bien que le Collectif existe, on se sent plus forte et enfin un peu écoutées, même si pas assez bien sûr.

En janvier dernier, on a beaucoup entendu parler de cette polémique autour des trente auteurs nommés au Grand Prix d'Angoulême, sans une seule autrice. Un gros pas en arrière, ou un reflet direct de la place des femmes dans le monde de la BD encore aujourd'hui?

Un reflet direct dans le sens qu'elles sont constamment mises au second plan. Les fe-

${ }^{4}$ Le Collectif des Créatrices de Bande Dessinée contre le Sexisme est un groupe d'autrices qui se sont réunies en décembre 2013, indignées d'être considérées comme des femmes avant de l'être comme des autrices de bande dessinée. Elles ont organisé plusieurs événements, comme le parodique «Les hommes et la BD». Bien qu'on pense souvent, dans le monde de la BD française, qu'il existe peu d'autrices, les signataires du Collectif sont plus de deux cents, et ont joué un rôle de premier plan lors du scandale du Grand Prix d'Angoulême en janvier 2016. Le collectif a un site web, http://bdegalite.org/, et publie régulièrement. La versión espagnole: http://bdegalite. org/english/carta-de-autoras/ 
plano. Las mujeres son muchas en el cómic, y sobre todo en la «nueva generación», pero seguimos siendo consideradas como anomalías. Para resumir, hace varios años que el festival permite a las autoras y autores votar para el Gran Premio, que distingue tanto el recorrido de un autor como su impacto en el mundillo del cómic - digo «autor» porque hasta ahora tan solo lo obtuvo una mujer, Florence Cestac-. Y bueno, desde que tiene lugar este voto público no se encontró entre los nominados más de dos mujeres (Marjane Satrapi y Posy Simmonds). ${ }^{5} \mathrm{Y}$ en 2016 tuvimos una sorpresa: no se encontró ni una sola autora. Ya hace varios años que algunas autoras se quejan de ello, en las redes sociales o en sus blogs, pero este año el Colectivo nos permitió tener audiencia, por fin, lo que creó una gran polémica en torno al Festival, y este contestó afirmando datos o hechos falsos. Es terrible que tengamos que llegar a semejante punto mientras hay tantas mujeres $y$ destacables en el cómic.

${ }^{5}$ Florence Cestac (Pont-Audemer, 1949) es una
autora de cómic francesa. En 1972 abrió la prime-
ra librería parisina de cómic y tres años más tarde
fundó la editorial Futuropolis. Es una de las autoras
más reconocidas, con varios premios desde 1989 en
Angoulême hasta 2014. Es una figura de excepción
en este sentido, ya que algunas autoras, como Clai-
re Brétécher, tienen una carrera tan larga y completa
como la suya pero nunca han sido distinguidas por el
Festival de Angoulême.
Marjane Satrapi es una autora franco-iraní, crea-
dora de la famosa novela gráfica Persépolis, publicada
en Francia entre 2000 y 2003 (en España entre 2002
y 2004) y adaptada al cine en 2007. Es autora tam-
bién de otras obras como Pollo con ciruelas, Bordados,
Los monstruos tienen miedo de la luna y El suspiro.

Posy Simmonds es una periodista, autora de novelas y de cómics e ilustradora de nacionalidad británica. Muchas de sus obras son conocidas por el público de masas, como Gemma Bovery y Tamara Drewe. Ha sido distinguida con varios premios en diferentes países, por ejemplo en España con el Premio Haxtur. mmes sont très nombreuses dans $1 \mathrm{a} \mathrm{BD}$, et surtout dans la «nouvelle génération», mais on continue à faire comme si on était des anomalies. Le festival a même menti pour se justifier, crétait vraiment un scandale. En gros, ça fait plusieurs années que le festival propose aux autrices et auteurs de voter pour le Grand Prix, qui est un prix qui récompense à la fois la carrière d`un auteur et son impact dans le monde de la BD — je dis «auteur» parce que seulement une femme, Florence Cestac, ${ }^{5}$ l'a eu-. Bref, depuis ce vote public, parmi les nominé-e-s on n'a jamais trouvé plus de deux femmes - Marjane Satrapi et Posy Simmonds—, et en 2016, on a eu la surprise de n'en trouver aucune. Ça fait plusieurs années que des autrices râlent sur les réseaux sociaux ou leur blog, mais cette année, la présence du Collectif a permis d'être enfin entendues, et ça a créé une grosse polémique autour du Festival, à laquelle celui-ci a répondu en mentant sur des faits ou des chiffres. C'est terrible d'en arriver là, quand les femmes sont si nombreuses et importantes dans la BD.

5 Florence Cestac (Pont-Audemer, 1949) est une autrice française de BD. En 1972, elle inaugura la première libraire de $\mathrm{BD}$ parisienne, et elle fonda trois ans plus tard les éditions Futuropolis. Elle fait partie des autrices les plus reconnues, avec plusieurs prix obtenus depuis 1989 à Angoulême jusqu'en 2014. En ce sens, elle fait figure d'exception : en effet, de nombreuses autrices, comme Claire Brétécher, ont un parcours aussi long et complet qu'elle, mais nont jamais été distinguées par le Festival d'Angoulême.

Marjane Satrapi est une autrice franco-iranienne, créatrice du fameux roman graphique Persépolis, publié en France entre 2000 y 2003 (entre 2002 y 2004 en Espagne) et adapté au cinéma en 2007. Elle est également l'autrice des oeuvres Poulet aux prunes, Broderies, Les Monstres n'aiment pas la lune, Le soupir, entre autres.

Posy Simmonds est une journaliste, autrice de romans et de BD et illustratrice, de nationalité britannique. Plusieurs de ses œuvres sont connues du grand public, comme Gemma Bovery et Tamara Drewe. Elle a été distinguée par plusieurs prix dans différents pays, par exemple en Espagne avec le prix Haxtur. 
Siendo autora, ¿has tenido entrevistas o reflexiones enfocadas al hecho de ser mujer cisexual ${ }^{6}$ en lugar de hablar de tu trabajo?

Tuve suerte, de todas mis entrevistas solo una o dos contenían preguntas tipo «bueno, hay que decir que lo que haces es muy femenino", lo que me dio mucha risa ya que por otra parte, en las críticas, casi siempre está escrito que dibujo mal. Eso sí, a lo mejor tiene que ver con mi género: aunque pueda entender que no guste mi estilo, que a mí personalmente me conviene perfectamente, estoy convencida de que si fuera un chico no me dirían tan a menudo que mi dibujo sea más «bonito».

En España hay un montón de autoras que abordan todos los estilos y temas posibles; ahora bien, se critican siempre las mismas publicaciones: el girly. ¿Qué opinas de este fenómeno en la edición francesa? ¿Presiona a las mujeres para que se dirijan hacia este tipo de identidad visual, criticándoles al mismo tiempo por ser fútiles?

Sí, totalmente: hubo muchos casos de plagio de autoras consideradas girly, y a veces cuando se expresaba la dibujante, explicaba que se lo habían pedido de manera explícita. Eso es lo que esperan de las autoras, pero después se permiten criticarles. Pénélope Bagieu $^{7}$ es un ejemplo muy relevante: tras

${ }^{6}$ Una persona cisexual tiene su identidad de género de acuerdo con el sexo que le ha sido atribuido al nacer. Se opone a las identidades transexuales, intersexuales... El cisexismo ignora deliberadamente estas identidades no-binarias y hace de la transexualidad una patología.

7 Pénélope Bagieu es una autora francesa que se hizo famosa en los últimos años gracias a su blog, publicado después en cómic. Tiene la reputación de
As-tu eu, en tant qu'autrice, des interviews / remarques centrées sur le fait que tu sois une femme cis ${ }^{6}$ avant de parler de ton travail?

J'ai eu de la chance, sur toutes mes interviews, seulement une ou deux contenaient des questions «alors c'est quand même très féminin ce que vous faites», ce qui m`a bien fait rigoler parce que le reste du temps, dans les critiques, il est quasi tout le temps écrit que je dessine mal. Drailleurs je pense que ça, ça a peut-être un lien avec mon genre: autant je peux comprendre qu'on n'apprécie pas mon style de dessin, qui me va personnellement très bien, autant je suis persuadée que si jétais un garçon, on me demanderait bien moins souvent d'avoir un dessin plus «joli».

En Espagne, il y a un paquet d'autrices qui abordent tous les styles graphiques et les thèmes possibles, or on critique toujours le girly. Qu'est-ce que tu penses de ce phénomène dans l'édition en France? Est-ce qu'on met la pression aux femmes pour se diriger vers ce genre d'identité visuelle, tout en les critiquant pour leur futilité?

Oui, complètement: il y a eu plein de cas de «plagiats» d'autrices dites girly et quand parfois la dessinatrice s'exprimait elle expliquait que c'est ce qu'on lui avait explicitement demandé. C'est ce qu'on attend des autrices, et ensuite on se permet de taper sur elle. Pénélope $\mathrm{Bagieu}^{7}$ en est un très bon exem-

${ }^{6}$ Una persona cisexual tiene su identidad de género de acuerdo con el sexo que le ha sido atribuido al nacer. Se opone a las identidades transexuales, intersexuales... El cisexismo ignora deliberadamente estas identidades no-binarias y hace de la transexualidad una patología.

${ }^{7}$ Pénélope Bagieu est une autrice française qui est devenue célèbre au cours des dernières années grâce à son blog, publié sur papier par la suite. Elle a la ré- 




LES VALEURS "FEMININES" RESULTENT DES PLACES OÚ ON ESSAYE DE CASER LES FEMMES:
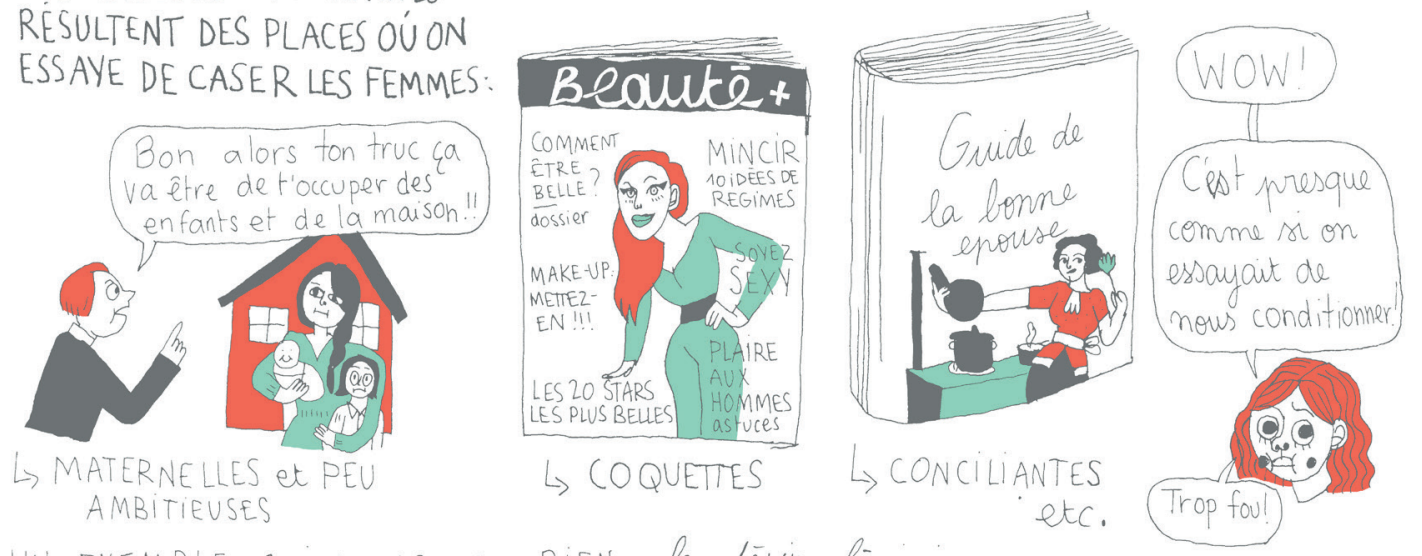

- UN EXEMPLE qUI LEMONTRE BIEN: le désir fiminin


Objetivo: desvalorizar a las mujeres. Las características «femeninas» despreciadas no son naturales sino adquiridas por la educación y los medios de comunicación.
Objectif: dévaloriser les femmes. Les caractéristiques «féminines» ne sont pas naturelles mais acquises par l'éducation et les médias. 


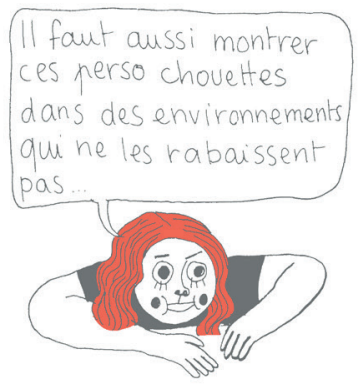

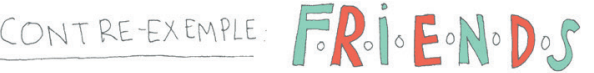

les 3 filles sont SUPER
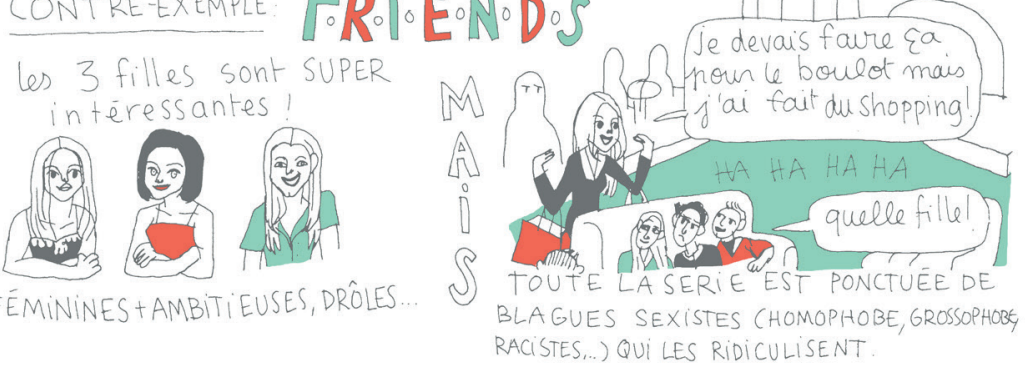

- Il EST AUSSI impORTANT DE DÉGENRER CES QUALITÉS!

$\rightarrow$ par exemple arec des perso féminins "virils"

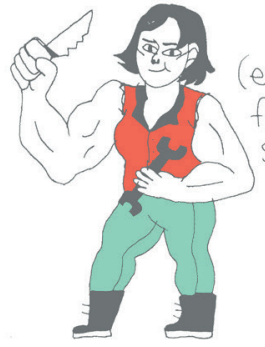

$\rightarrow$ mais aussi avec des
perso masculins "féminin
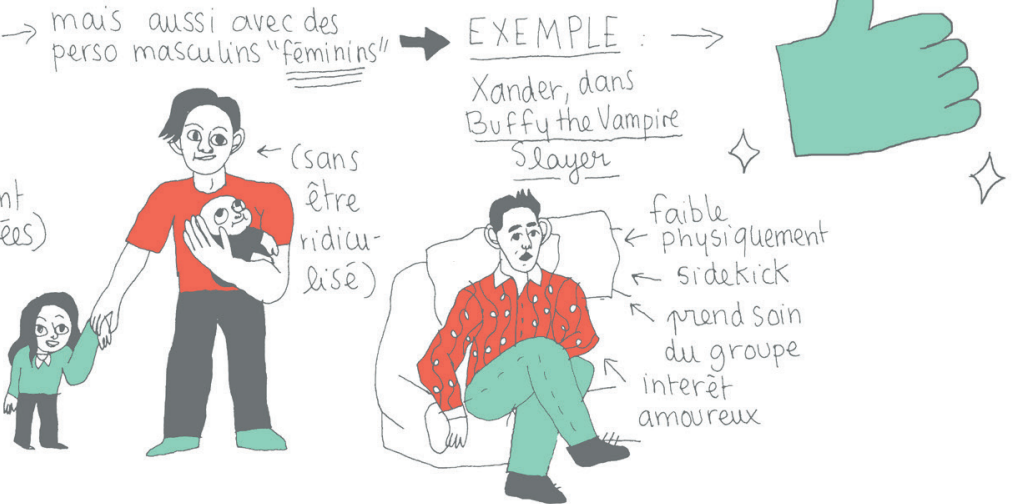

\section{Conclusion:}

ON A BESOIN DES VALEURS "FEMININES"
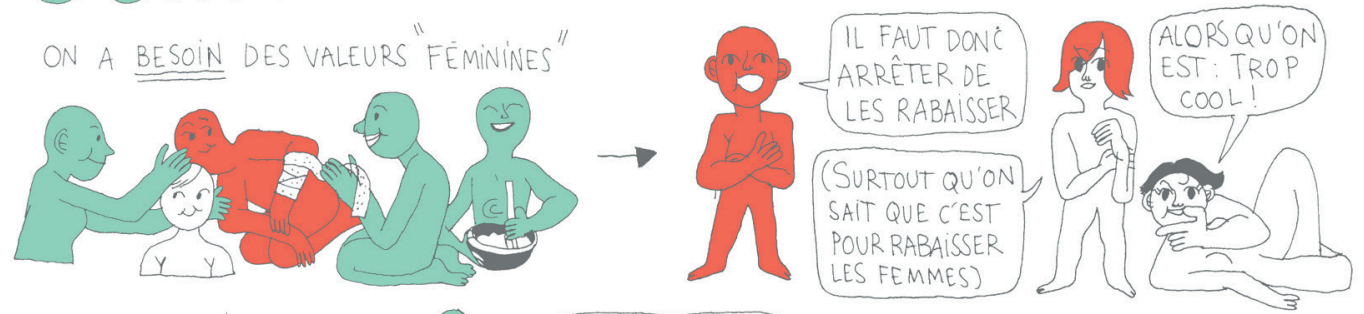

LA FORCE PHYSIQUE N'EST PAS LA SEULE BONNE QUALITE POSSIBLE
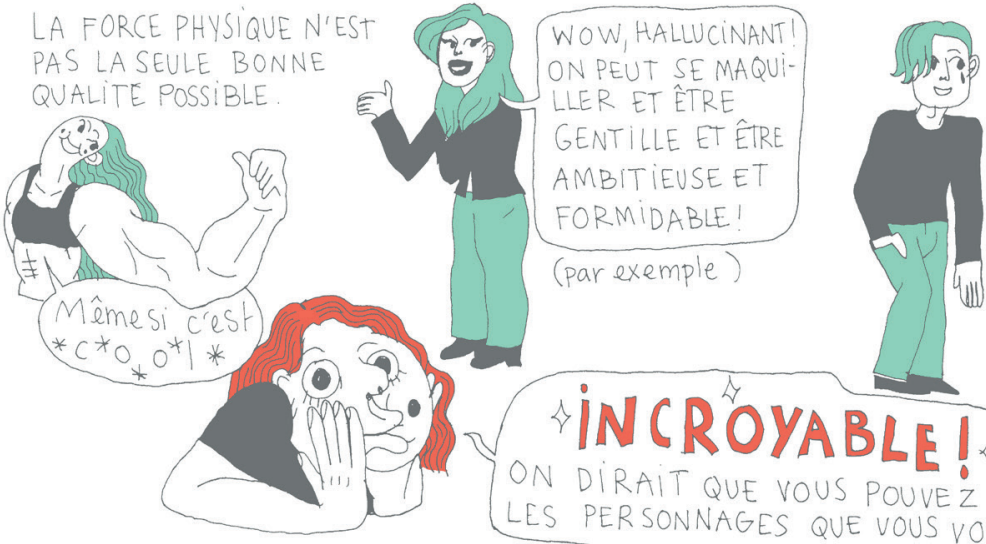



(par exemple encore)

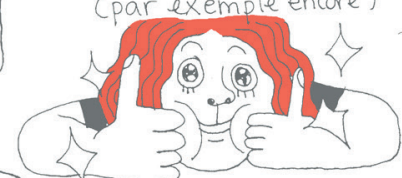

$\square ? \stackrel{0}{\mathrm{~L}}$
Apoyada en el ejemplo de la serie Friends, Mirion pone de realce la importancia de construir personajes con matices, sin distinción de género.
Dans cette page, Mirion souligne l'importance de construire des personnages complexes sans distinction de genre, prenant comme exemple la série Friends. 
haber empezado con un estilo que fue considerado girly, en el cual hablaba de zapatos, sí, pero también simplemente de su vida cotidiana y de muchos temas «neutros». Ahora ha publicado un montón de libros - que además se enfocan muchas veces a heroínas y es genial- Son muy buenos, es en este momento una de las autoras más innovadoras. Y en paralelo, todavía veo mucha gente, incluso del medio del cómic, burlarse de ella y reducirla a «cupcakes $\&$ macarons». ${ }^{8}$ Eso me mata, representa bien la manera de pensar que hay en este medio.

\section{¿Tienes nuevos proyectos en mente? ¿Y al- gunos consejos para los futuros autores $y$ autoras?}

Pues sí: muchos proyectos de cómic que me cuesta poder empezar, por ejemplo un libro con una amiga para criticar los consejos de seducción tan nocivos que se dan a los adolescentes. También me gustaría mucho volver a la ficción, jtengo dos proyectos de historias que querría continuar!

En cuanto a los consejos: dibujar, enseñar lo que hacéis al máximo de gente que podáis y pedir críticas, pasarlo bien y hacer lo que os apetezca. Y sobre todo, no dejar que gane la gente que os insulta gratis, sobre todo a las mujeres: sois vosotras las más fuertes.

abordar temáticas específicamente femeninas, aunque escribió varias novelas gráficas de todo tipo y nunca se quiso meter en la franja girly, que es más bien una categoría comercial de los editores. Les culottées, el blog de Pénélope Bagieu en el periódico Le Monde, destaca cada semana la vida de una mujer que marcó la historia: http://lesculottees.blog. lemonde.fr/

${ }^{8}$ Pasteles muy de moda en París, tópico de la parisina femenina y elegante. ple: après avoir commencé dans un style qu'on a nommé girly, où certes elle parlait de chaussures mais aussi tout simplement de sa vie quotidienne et de plein de sujets «neutres», elle a maintenant tout un tas d'albums derrière elle — qui sont d'ailleurs souvent centrés sur des héroïnes et c'est pas mal super, qui sont très bons, c'est une des autrices les plus innovantes du moment-. Mais à côté, je vois toujours plein de gens, notamment du milieu de la BD, se moquer d'elle et la ramener à «cupcakes et macarons». Ça me tue, ça montre bien l'état d'esprit du milieu.

Des idées pour la suite? Des conseils à donner aux futurs auteurs et surtout aux autrices?

Alors oui: plein de projets de BD que j'ai hâte de pouvoir commencer, par exemple un livre avec une amie pour critiquer les conseils de drague très nocifs qu'on donne aux adolescents. J'aimerais beaucoup aussi me remettre à la fiction, j'ai deux projets d'histoire que je voudrais continuer!

Et les conseils: dessiner, montrer ce qu'on fait à un maximum de gens et demander des critiques, s'amuser et faire ce dont on a envie. Et surtout, ne pas laisser gagner les gens nuls qui insultent gratuitement, surtout pour les femmes: vous êtes bien les plus fortes.

putation d'aborder des thématiques spécifiquement féminines, alors qu'elle a réalisé plusieurs romans graphiques de tout genre et n'a jamais revendiqué l'identité girly, qui correspond plutôt à une catégorie commerciale des éditeurs. Les culottées, le blog de Pénélope Bagieu publié dans Le Monde, raconte chaque semaine la vie d'une femme qui a marqué l'histoire: http://lesculottees.blog.lemonde.fr/ 